\title{
Syntrophic growth of alkaliphilic anaerobes controlled by ferric and ferrous minerals transformation coupled to acetogenesis
}

\author{
Daria G. Zavarzina ${ }^{1} \cdot$ Sergey N. Gavrilov ${ }^{1} \cdot$ Natalia I. Chistyakova $\mathbb{C}^{2} \cdot$ Angelina V. Antonova $\mathbb{C}^{2} \cdot$ \\ Maria A. Gracheva $\mathbb{D}^{2} \cdot$ Alexandr Yu. Merkel ${ }^{1} \cdot$ Anna A. Perevalova ${ }^{1} \cdot$ Michail S. Chernov $^{3} \cdot$ Tatyana N. Zhilina $^{1} \cdot$ \\ Andrey Yu. Bychkov ${ }^{3}$ - Elizaveta A. Bonch-Osmolovskaya ${ }^{1,4}$
}

Received: 29 April 2019 / Revised: 2 August 2019 / Accepted: 14 September 2019 / Published online: 22 October 2019

(c) The Author(s), under exclusive licence to International Society for Microbial Ecology 2019

\begin{abstract}
Redox-active iron minerals can act as energy sources or electron-transferring mediators in microbial syntrophic associations, being important means of interspecies metabolic cooperation in sedimentary environments. Alkaline conditions alter the thermodynamic stability of iron minerals, influencing their availability for interspecies syntrophic interactions. We have modeled anaerobic alkaliphilic microbial associations in ethanol-oxidizing co-culture of an obligate syntroph Candidatus "Contubernalis alkalaceticum" and a facultative lithotroph Geoalkalibacter ferrihydriticus, which is capable of dissimilatory $\mathrm{Fe}(\mathrm{III})$ reduction and homoacetogenic oxidation of $\mathrm{Fe}(\mathrm{II})$ with $\mathrm{CO}_{2}$. The co-cultures were cultivated with thermodynamically metastable ferric-containing ferrihydrite, or ferrous-containing siderite, or without minerals. Mössbauer spectral analysis revealed the transformation of both minerals to the stable magnetite. In the presence of ferrihydrite, G. ferrihydriticus firstly reduced $\mathrm{Fe}(\mathrm{III})$ with ethanol and then switched to syntrophic homoacetogenesis, providing the growth of obligate syntroph on ethanol. The ability of G. ferrihydriticus to accept hydrogen from its syntrophic partner and produce extra acetate from carbonate during ethanol oxidation was confirmed by co-culture growth without minerals. In the presence of siderite, G. ferrihydriticus performed homoacetogenesis using two electron donors simultaneously- siderite and hydrogen. Pieces of evidence for direct and indirect hydrogen-mediated electron exchange between partner organisms were obtained. Relative abundancies of partner organisms and the rate of acetate production by their co-cultures were strongly determined by thermodynamic benefits, which G. ferrihydriticus got from redox transformations of iron minerals. Even the minor growth of G. ferrihydriticus sustained the growth of the syntroph. Accordingly, microbe-to-mineral interactions could represent underestimated drivers of syntrophic interactions in alkaline sedimentary environments.
\end{abstract}

These authors contributed equally: Sergey N. Gavrilov, Natalia I. Chistyakova, Angelina V. Antonova

Supplementary information The online version of this article (https:// doi.org/10.1038/s41396-019-0527-4) contains supplementary material, which is available to authorized users.

\section{Daria G. Zavarzina}

zavarzinatwo@mail.ru

1 Winogradsky Institute of Microbiology, Federal Research Center of Biotechnology, Russian Academy of Sciences, Prospekt 60 Letiya Oktyabrya 7, building 2, Moscow, Russian Federation 117312

2 Department of Physics, Lomonosov Moscow State University, Leninskie Gory 1, Moscow, Russian Federation 119991

3 Department of Geology, Lomonosov Moscow State University, Leninskie Gory 1, Moscow, Russian Federation 119991

4 Department of Biology, Lomonosov Moscow State University, Leninskie Gory 1-12, Moscow, Russian Federation 119991

\section{Introduction}

Interspecies redox interactions play a fundamental role in the process of organic matter digestion and act as the driving force for its complete conversion to $\mathrm{CO}_{2}$ and $\mathrm{CH}_{4}$ through a series of metabolic reactions [1]. Indirect interspecies electron transfer (IIET) via hydrogen occurring in syntrophic associations was discovered more than 50 years ago [2]. Since then to now, it is intensively studied (see refs. [3-5] for review). Hydrogen and formate are the most important electron carriers between syntrophic partners in IIET, but besides them, this function was reported for anthraquinone-2,6-disulfonate, cysteine, or inorganic sulfur compounds [6-9]. Although methanogens are the most widely studied hydrogen-utilizing members of syntrophic associations, those with hydrogenotrophic sulfate- or ironreducing bacteria have also been demonstrated [10-12]. 
Direct interspecies electron transfer (DIET) as an alternative to IIET was first reported in the co-culture of iron-reducing bacteria Geobacter metallireducens and Geobacter sulfurreducens [13]. It was assumed that DIET involves $\mathrm{Fe}(\mathrm{III})$-reducing or $\mathrm{Fe}$ (II)-oxidizing organisms and is realized through biological electrical connections via multiheme $c$-type cytochromes and electrically conductive pili [14], or through abiotic connections via conductive materials such as magnetite [15].

It is known that $\mathrm{pH}$ may affect microbial metabolism and hence microbial community structures by modulating the thermodynamics and kinetics of redox reactions. In particular, the available energies of syntrophic oxidation reactions increase with increasing $\mathrm{pH}[16,17]$. However, despite intensive studies of IIET and DIET at neutral conditions, the information on this type of microbial cooperation in alkaline environments (such as soda lakes) is very limited. To date, two alkaliphilic acetate-oxidizing syntrophic associations isolated from soda lakes were described $[18,19]$, and in both of them, the bacteria of the family Syntrophomonadaceae were identified. Candidatus "Contubernalis alkalaceticum" oxidizes acetate, ethanol, and some other organic compounds in co-cultures with haloalkaliphilic hydrogenotrophic sulfate-reducing bacteria (SRB) of the genus Desulfonatronum [18, 20], while Candidatus "Syntrophonatronum acetioxidans" oxidizes only acetate in association with a hydrogenotrophic partner of the genus Desulfonatronospira [19]. Both Syntrophomonads are obligate syntrophs, and all attempts to isolate them in pure cultures were unsuccessful. In 2016, Sorokin et al. [21] described three new genera of obligate syntrophs of the order Syntrophomonadales able to oxidize straight-chain fatty acids in association with alkaliphilic methanogens of the genus Methanocalculus or alkaliphilic SRB. A methanogenic association containing obligate syntrophic bacteria closely related to $C$. "C. alkalaceticum" (99\% $16 \mathrm{~S}$ ribosomal RNA (rRNA) gene sequence similarity) was also described. Thus, this microorganism was shown to participate in syntrophic association with different hydrogenutilizing partners [21].

Over the past decade, several alkaliphilic dissimilatory iron-reducing bacteria able to reduce synthesized ferrihydrite were isolated (see references in ref. [22]). Among them, Geoalkalibacter ferrihydriticus was shown not only to reduce $\mathrm{Fe}$ (III) but also to oxidize $\mathrm{Fe}$ (II) during anaerobic biotransformation of iron-containing micas [22, 23]. Electrochemical and chronoamperometric studies showed that G. ferrihydriticus possesses diverse pathways for anode respiration [24], and can perform long-range direct electron transfer through electrode-attached biofilms in a microbial fuel cell without soluble electron shuttles [25, 26]. These features, as well as the ability of the organism to oxidize hydrogen as an electron donor [27], make it the perfect candidate to perform interspecies electron transfer in alkaline microbial communities.

The aim of this work was to investigate the modes of syntrophic interactions between obligately syntrophic organotroph C. "C. alkalaceticum" and iron-cycling G. ferrihydriticus, capable of lithoautotrophic and heterotrophic growth. We report the results obtained with two iron minerals, in which iron was present at different oxidation states: Fe(III) —-ferrihydrite, and Fe(II)_-siderite. Syntrophic culture lacking any forms of iron, with carbonate as the only terminal electron acceptor, was used as a control.

\section{Materials and methods}

\section{Bacterial strains and minerals}

In this work, the type species of $C$. "C. alkalaceticum" Z$7904^{\mathrm{T}}\left(=\mathrm{VKM} \mathrm{B}-2362^{\mathrm{T}}\right)$ and G. ferrihydriticus $\mathrm{Z}-0531^{\mathrm{T}}$ $\left(\mathrm{DSMZ}=17461^{\mathrm{T}}=\right.$ VKM B-2349 $\left.{ }^{\mathrm{T}}\right)$ were used [18, 23]. To get rid of the hydrogen-utilizing partner, the initial syntrophic culture, containing the spore-forming $C$. "C. alkalaceticum" and the thermolabile SRB Desulfonatronum cooperativum $\mathrm{Z}-7999^{\mathrm{T}}\left(\mathrm{DSMZ}=16749^{\mathrm{T}}=\mathrm{VKM}\right.$ B-2329 ${ }^{\mathrm{T}}$ ) [21], was heated at $100^{\circ} \mathrm{C}$ for $10 \mathrm{~min}$. Remaining culture containing only spores of $C$. "C. alkalaceticum" was mixed with a pure culture of $G$. ferrihydriticus. The absence of $D$. cooperativum in this artificially combined culture was confirmed by new generation sequencing (NGS) of $16 \mathrm{~S}$ rRNA gene fragments. Cell morphology of syntrophic partners differed from each other. Cells of $C$. "C. alkalaceticum" are straight or slightly curved nonmotile rods of $0.5-0.7 \times 2.0-7.0 \mu \mathrm{m}$, forming terminal endospores [18]. Cells of $G$. ferrihydriticus are short, motile non-sporeforming rods of $0.3-0.5 \times 1.0-2.0 \mu \mathrm{m}$ [23]. Morphological differences were steadily reproduced in all our experiments and allowed us to assess the relative abundance of both organisms in the co-cultures by direct cell counting. The obtained data correlated with the results of 16S rRNA gene fragments profiling of the co-cultures performed at the final points of all the experiments.

Ferrihydrite, synthesized as described previously [23], was presented in nanoparticles [28]. Selected grains of hydrothermal siderite $\mathrm{FeCO}_{3}$ (Bakal deposit, Ural, Russia) were crushed into powder $(<100 \mu \mathrm{m}$ particle size). Its purity was confirmed by X-ray spectroscopy, which revealed no detectable content of secondary minerals.

\section{Cultivation}

The combined cultures of $C$. "C. alkalaceticum" and G. ferrihydriticus were inoculated to a medium containing $25 \mathrm{mM}$ of ethanol as the substrate for $C$. "C. alkalaceticum" 
with or without ferrihydrite. No stable growth of the coculture on the same medium with acetate was observed in preliminary tests.

For all experiments, we used anaerobically prepared media with the following composition $\left(\mathrm{g}^{-1}\right): \mathrm{KH}_{2} \mathrm{PO}_{4}-0.2$; $\mathrm{MgCl}_{2}-0.1 ; \mathrm{NH}_{4} \mathrm{Cl}-0.5 ; \mathrm{KCl}-0.2 ; \mathrm{NaCl}-1.0 ; \mathrm{Na}_{2} \mathrm{CO}_{3}-3.0$; $\mathrm{NaHCO}_{3}-10.0$; yeast extract-0.05; trace element solution [29]-1 $\mathrm{ml} \mathrm{l}^{-1}$; ethanol-25 or $35 \mathrm{mM}$ for the experiments with and without minerals, respectively. The media were dispensed under $\mathrm{N}_{2}$ flux by $30-\mathrm{ml}$ portions into $60-\mathrm{ml}$ glass bottles, each containing ferrihydrite (final content of $\mathrm{Fe}$ atoms $75 \mathrm{mM}$ ) or siderite (final content of $\mathrm{Fe}$ atoms $25 \mathrm{mM}$ ), or no minerals, and were autoclaved at $120^{\circ} \mathrm{C}$ for $30 \mathrm{~min}$.

The media were inoculated with the co-culture of $C$. "C. alkalaceticum" and G. ferrihydriticus (5\% vol). All kinetic experiments were performed in triplicate. For each experiment, controls with sterile media, as well as with the media inoculated with the pure culture of G. ferrihydriticus or the co-culture and further heat-treated at $100{ }^{\circ} \mathrm{C}$ for 10 min, were set-up.

\section{Analytical methods}

Bacterial growth, the concentrations of ethanol, acetate, and $\mathrm{Fe}(\mathrm{II})$ (for the experiment with ferrihydrite), was monitored during all the incubation periods. As cells of two bacterial lineages clearly differed from each other by their morphology, direct cell counting was used for the assessment of cell growth with a phase contrast light microscope (Reichert Zetopan, Austria). Epifluorescence microscopy was performed to assess the growth on mineral particles: $2 \mu \mathrm{l}$ of agitated culture samples were dried on the slide, routinely stained with acridine orange and studied with an AxioImager.D1 microscope (Carl Zeiss, Germany), which was equipped with relevant filters (Zeiss 20 for Cy3-labeled probes and Zeiss 49 for DAPIstained cells).

Fermentation products were determined by gas chromatography. Hydrogen was assayed using a Crystal 5000.2 gas chromatograph (Chromatec, Russia) equipped with a thermal conductivity detector and a 1-m column filled with Carboxen-1000 column, 60-80 mesh (Supelco), all conditioned isothermally at $140{ }^{\circ} \mathrm{C}$ with argon as the carrier gas. Acetate and ethanol were assayed using the same chromatograph with the FID detector and capillary column Optima FFAPplus $0.25 \mu \mathrm{m} \times 0.32 \mathrm{~mm} \times 30 \mathrm{~m} \quad$ (Macherey-Nagel) with argon as the carrier gas. Separation was carried out with temperature programming. Samples for chromatography $(0.2 \mathrm{ml})$ were pre-treated by centrifugation at $12600 \times g$ for $2 \mathrm{~min}$, followed by the acidification of clear supernatants with $5 \mathrm{M}$ formic acid to the $\mathrm{pH}$ 2.0. Detection limit of the method was $0.2 \mathrm{mM}$.
Precipitates of ferrihydrite formed after centrifugation were dissolved in $6 \mathrm{~N} \mathrm{HCl}$ and the $\mathrm{Fe}(\mathrm{II})$ content was determined colorimetrically with ferrozine [30].

The mineral composition was studied by using X-ray diffractometry on the Panalytical Empyrean diffractometer using a copper anode $(\lambda=1.54 \AA$, operating modes $I=40$ $\mathrm{mA}, U=40 \mathrm{kV}$ ) and a Pixel3D two-coordinate detector and by Fourier-transform infrared spectroscopy (Nicolet 6700 $\mathrm{XT}$, Thermo Electron Corp.) with the $\mathrm{KBr}$ pellet technique.

Mössbauer spectroscopy was applied in accordance with the following protocol. Spectra of ${ }^{57} \mathrm{Fe}$ nuclei were recorded at $T=82,295 \mathrm{~K}$ on an MS-1104Em, which was operated in a constant acceleration mode with a ${ }^{57} \mathrm{Co}$ source in the $\mathrm{Rh}$ matrix. The calibration was given relatively to $\alpha$-Fe. The SpectrRelax software was used to process and analyze the Mössbauer spectra [31].

The microstructure of the samples was routinely studied by scanning electron microscopy (SEM) on LEO 1450VP (Carl Zeiss, Germany).

\section{Thermodynamic calculations}

Equilibrium and metastable states of the system $(\mathrm{H}-\mathrm{C}-\mathrm{O}-\mathrm{Na}-\mathrm{Cl}-\mathrm{Fe})$ were calculated using the HCh software $[32,33]$. To calculate the equilibrium state, the minimum free energy of the system was determined. Excess energy for metastable state was estimated by the reaction path model [34], and the reaction progress (extent of reaction) was calculated by content of acetate, ethanol, and $\mathrm{Fe}$ (II), measured in the experiments. Changes in Gibbs-free energy of reactions were calculated using the HCh software, and thermodynamic properties of components were taken from the Unitherm database [32]. To calculate $\Delta G_{r}^{\exp }$, the values for the standard state $\left(\Delta G_{r}^{\circ}\right)$ were recalculated for real $\mathrm{pH}$ values, bicarbonate activity. The value of $0.2 \%$ $\mathrm{H}_{2}\left(P_{\mathrm{H}_{2}}=0.002\right.$ bar $)$ was used. For reaction with acetate as a substrate, its concentration was taken as $25 \mathrm{mmol}$. For reactions of incomplete ethanol oxidation, the concentrations of ethanol and acetate were taken as equal. These values were used to estimate the direction of reactions under experimental conditions.

\section{High-throughput sequencing of 16S rRNA gene fragments}

DNA samples from the cell pellets formed after centrifugation were isolated as described previously [35]. The libraries were prepared using polymerase chain reaction with universal primers to the V3-V4 region of 16S rRNA gene in accordance with the previously described technique [36]. The following primer system was used: Pro-mod-341F (5'- CCTAYGGGDBGCWSCAG-3') and Pro-mod-805R 5'-GACTACNVGGGTMTCTAATCC-3' [37]. Sequencing 

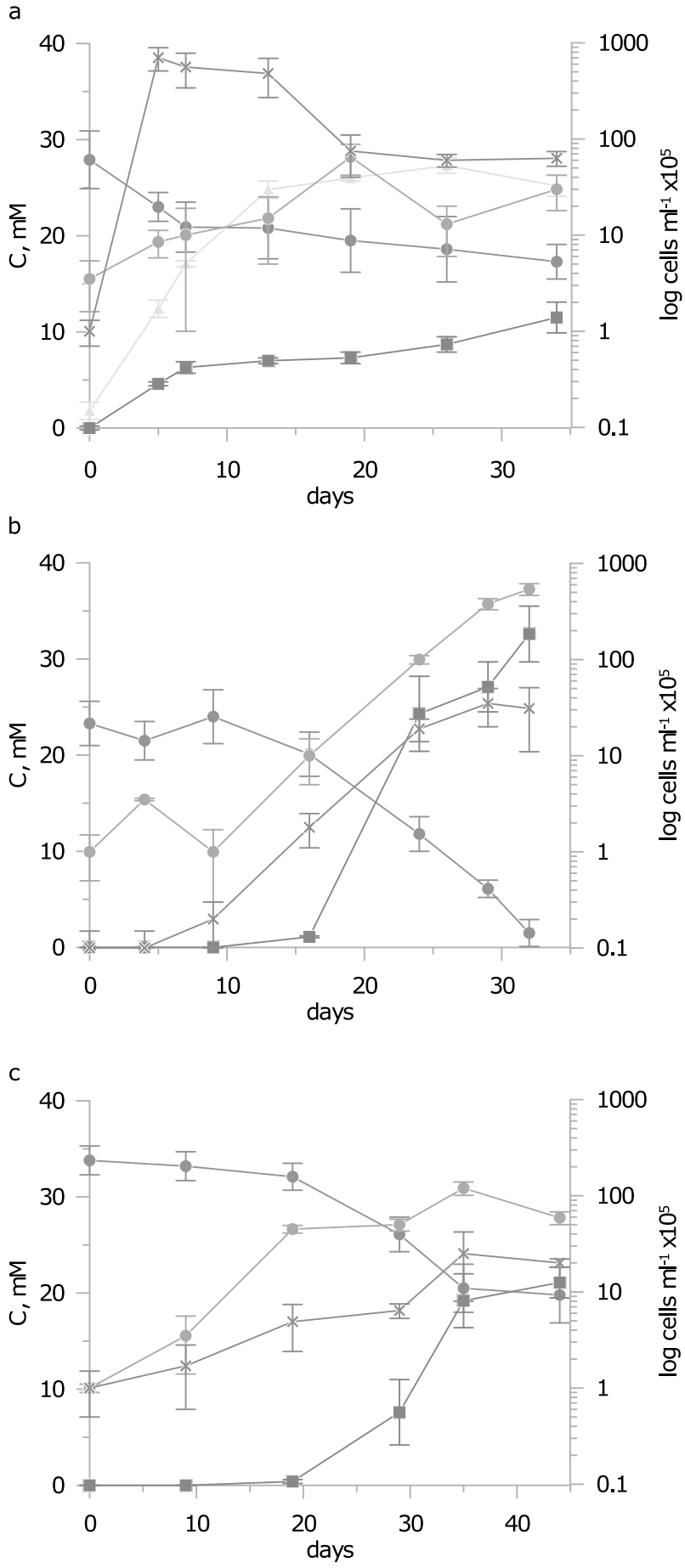

Fig. 1 Growth of C. "C. alkalaceticum" and G. ferrihydriticus on the medium with (a) ferrihydrite; (b) siderite; (c) without iron minerals. $N=3$. Legend: blue circles - ethanol; red squares-acetate; yellow triangles (a)-Fe(II); purple crosses-G. ferrihydriticus; lightgreen circles circles-C. "C. alkalaceticum"

was carried out on a MiSeq system (Illumina, USA) using the reagent kit, which can read 300 nucleotides from each end. Demultiplexing, as well as subsequent treatment and analysis of the sequences, was carried out using the relevant scripts of the QIIME ver. 1.9.0 software package [38]. The OTU table was constructed using the SILVAngs online service (www.arb-silva.de/ngs). All the sequencing data are deposited in NCBI BioProject PRJNA556462.

\section{Genome analysis}

For genome analysis, the tools of the IMG/ER server (https://img.jgi.doe.gov/cgi-bin/mer/main.cgi) were used. Sequences used as templates for specific gene searches are described in the Results section. The G. ferrihydriticus genome version from the IMG database (Genome ID 2599185148) was used for analysis. Genes from the xap operon (xapABCDEFGHIJ) were identified with characteristic motifs as previously described [39].

\section{Results}

\section{Growth of C. "C. alkalaceticum" and G. ferrihydriticus co-culture in the presence of ferrihydrite}

Ethanol is the substrate for both members of the syntrophic co-culture studied, though for its utilization G. ferrihydriticus needs ferrihydrite, and $C$. "C. alkalaceticum" needs the presence of a hydrogen-utilizing partner. Thus, regarding the medium with ethanol and ferrihydrite, we could expect the competition of two processes - organotrophic or/and hydrogenotrophic iron-reduction, both with the participation of G. ferrihydriticus.

Active growth of $G$. ferrihydriticus on the medium with ferrihydrite started after 3 days of incubation and was accompanied by a change in the precipitate color from brown to brownish-black, which indicates ferrihydrite reduction to magnetite. Cells of G. ferrihydriticus dominated during the first 13 days of incubation. Within this period, active reduction of ferrihydrite, accompanied by ethanol oxidation and the formation of an equivalent amount of acetate, was registered (Fig. 1a and Supplementary Table 1). C. "C. alkalaceticum" growth started after the 10th day of incubation, when the main part of ferrihydrite was reduced by $G$. ferrihydriticus. During the last 11 days, on average, $3.5 \mathrm{mM}$ of ethanol were utilized and $4.5 \mathrm{mM}$ of acetate were produced, while $\mathrm{Fe}(\mathrm{II})$ concentration practically did not change during this period (Fig. 1a and Supplementary Table 1). Light and epifluorescence microscopy revealed the prevalence of $G$. ferrihydriticus in the co-culture within the first 13 days of incubation. Further decrease in $G$. ferrihydriticus cell counts (Fig. 1a) was caused by the active colonization of mineral particles (Supplementary Fig. 1). By the end of incubation, NGS sequencing of 16S rRNA gene fragments retrieved the ratio of $C$. "C. alkalaceticum" to G. ferrihydriticus cells around 

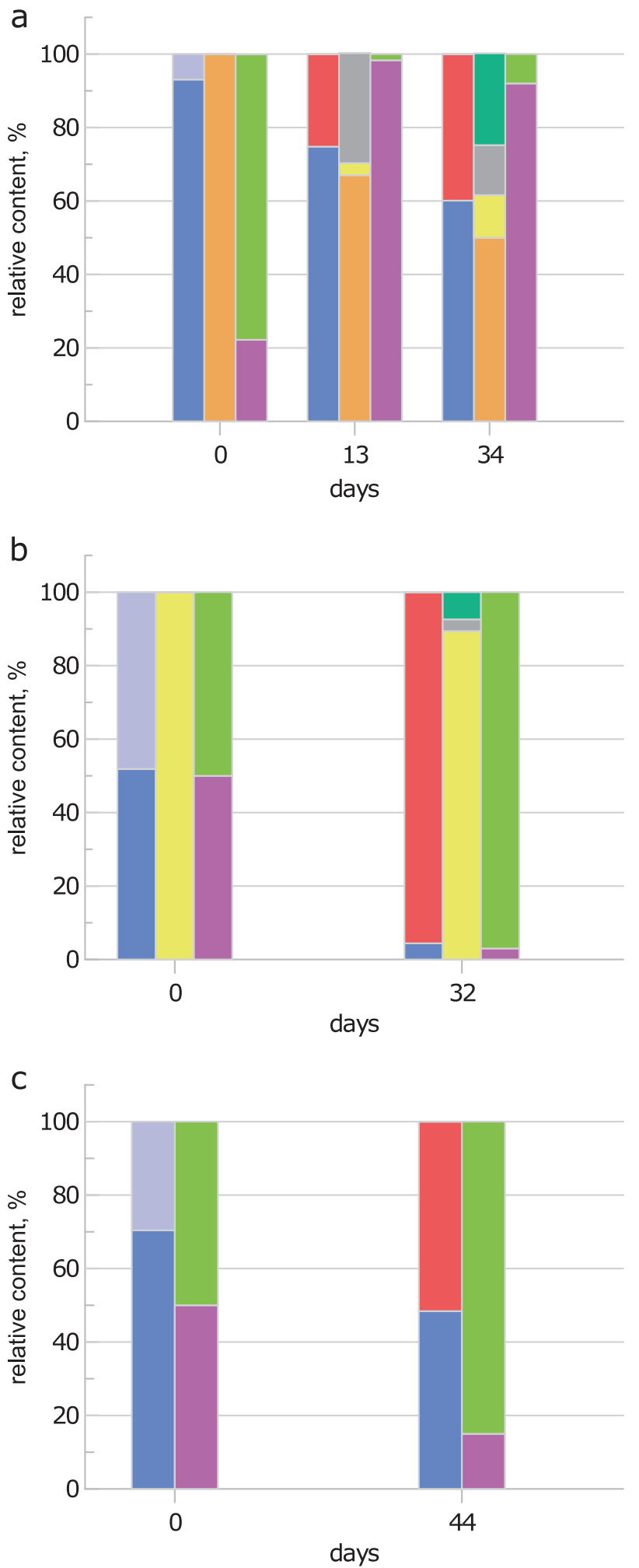

1:11 (Fig. 2a, last column). Thus, in the presence of ferrihydrite, $C$. "C. alkalaceticum" and G. ferrihydriticus compete with each other for ethanol, and syntrophic growth starts only when ferric iron, covered with reduced product, was not available anymore. G. ferrihydriticus switches to
Fig. 2 Graphical summary of the results of growth experiments with ferrihydrite (a), siderite (b), and without minerals (c). All data are expressed in percent. Legend. First column in each group-carbon substrate to product ratio: blue-ethanol; red-acetate; light-grayamount of carbonate consumed via lithotrophic acetogenesis, as calculated from the amount of acetate produced. Second column in each group-relative content of iron minerals: orange—ferrihydrite; yellow — siderite; darck-gray-magnetite; blue-green—green rust. Third column in each group-relative abundance of syntrophic partner bacteria as calculated from the results of $16 \mathrm{~S}$ rRNA gene fragments profiling: light-green-C. "C. alkalaceticum"; purple-G. ferrihydriticus

hydrogenotrophic metabolism as a partner of $C$. "C. alkalaceticum", producing an additional amount of acetate from $\mathrm{CO}_{2}$.

Mössbauer analysis of samples collected after 5 days of incubation under the prevalence of $G$. ferrihydriticus demonstrated that ferrihydrite reduction led to the formation of small particles of a magnetite-maghemite mixture $(30 \%)$ and siderite $(3 \%)$. The spectrum of this sample obtained at $82 \mathrm{~K}$ is presented in Fig. 3a. The model consisting of two doublets and a relaxation-type sextet was used to process the spectrum. Doublet $D_{1}$ corresponds to ferric atoms in the ferrihydrite structure, $D_{2}$-ferrous atoms in a siderite structure; relaxation-type sextet $S$ is associated with small particles of magnetite-maghemite mixture (Fig. 3a). The same analysis performed after the end of microbial growth showed that $16 \%$ of magnetite that was formed earlier underwent significant transformations during the culture growth. The spectra of these samples (Fig. 3b) were more complicated and fitted with five doublets and a relaxationtype sextet. $D_{1}, D_{2}$, and $S$ can be interpreted the abovementioned way. Three extra doublets added to the fitting model most probably could be interpreted as green rust, where doublets $D_{3}$ and $D_{4}$ correspond to ferrous and $D_{5}$ - to ferric atoms, respectively. No structural changes of ferrihydrite appeared in the sterile control (Fig. 3c). A relative intensity of biogenic mineral phases is presented in Fig. 3a, the middle column, and in Supplementary Table 4.

No ethanol oxidation, acetate accumulation, or ferrihydrite transformation was observed in the control experiment with the heat-treated co-culture of $C$. "C. alkalaceticum" and G. ferrihydriticus. The pure culture of G. ferrihydriticus grew on the medium with ethanol and ferrihydrite oxidizing $6.8 \pm$ $0.1 \mathrm{mM}$ ethanol, producing $20 \pm 0.5 \mathrm{mM}$ of Fe (II) and $6.5 \pm$ $0.2 \mathrm{mM}$ acetate, and, thus, repeating our previous results [27].

\section{Growth of C. "C. alkalaceticum" and G. ferrihydriticus co-culture on the medium with siderite}

Siderite is a carbonate mineral containing only $\mathrm{Fe}(\mathrm{II})$ atoms. The evidence on the ability of $G$. ferrihydriticus to grow anaerobically with ferrous iron as an electron donor [22] 

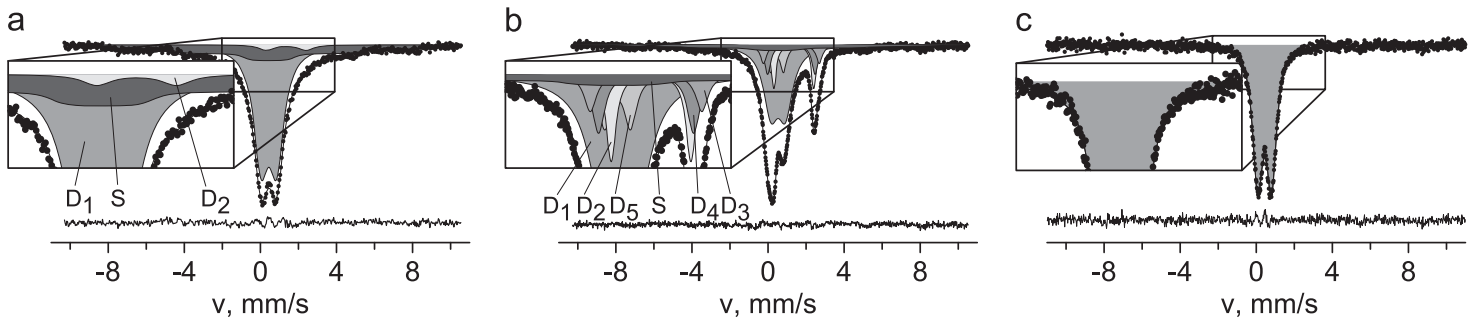

Fig. 3 Mössbauer spectra of iron minerals measured at $82 \mathrm{~K}$ for the experiment with ferrihydrite. Samples taken at: (a) 5th day of incubation; (b) 34th day of incubation; (c) from sterile control at the end of incubation
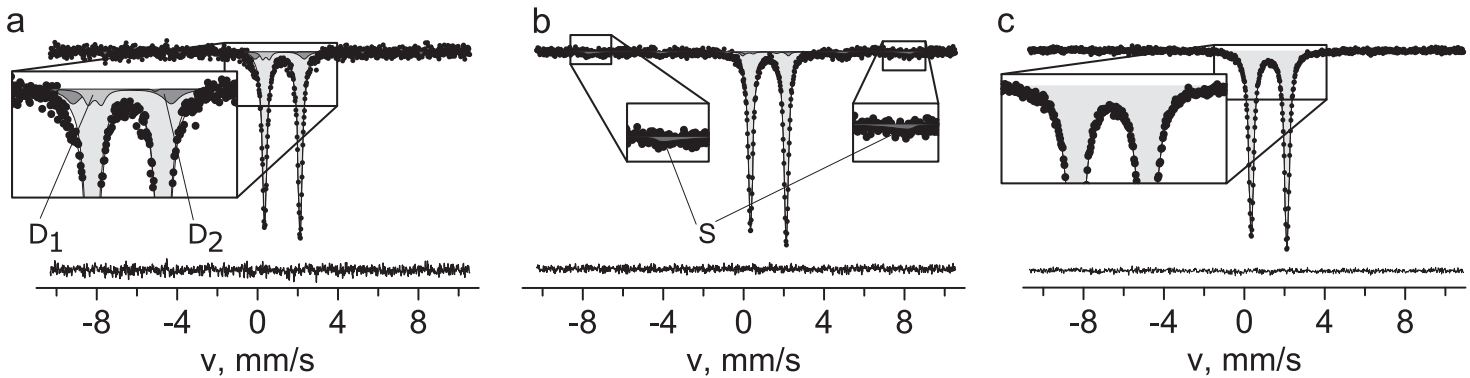

Fig. 4 Mössbauer spectra of iron minerals measured at room temperature for the experiments with siderite. All samples were taken at the end of incubation, (a)—from the 1st culture replicate; (b)—from the 2nd culture replicate; (c) —from sterile control

inspired us to test the growth of a syntrophic co-culture with this mineral. In this case, two electron donors (ethanol and siderite) were present in the medium simultaneously, while carbonate was the only electron acceptor.

The start of the C. "C. alkalaceticum" and G. ferrihydriticus co-culture growth on the medium with siderite was registered by the changes in siderite color from orangebrown to dark-gray (Supplementary Fig. 2). Lag phase in different replicates lasted from 16 to 40 days, but further growth phases were of similar duration in all the replicates. The results presented in Supplementary Table 2 were normalized for the most rapid lag phase. In all the cultures, siderite color change was accompanied by ethanol oxidation, acetate formation, and increase in cell concentration of both microorganisms. In liquid phase, the number of $C$. " $\mathrm{C}$. alkalaceticum" and G. ferrihydriticus cells started to increase simultaneously, with the constant ratio 10:1 during all the incubation time (Fig. 1b). C. "C. alkalaceticum" formed chains of 3-6 cells each, which were often attached to siderite particles (Supplementary Fig. 3a). Approximately, the same ratio of two microorganisms was observed by epifluorescent microscopy after 1 month of incubation. The results of 16S rRNA gene fragment profiling after the end of incubation showed the ratio between $C$. "C. alkalaceticum" and G. ferrihydriticus cells of 32:1 (Fig. 2b last column in the groups), which, in general, supported direct cell-counting results. At the end of the experiment,
$21.8 \pm 1.4 \mathrm{mM}$ ethanol (95\% of initial amount; Fig. 2b, first columns in the groups) were consumed and $32.6 \pm 2.9 \mathrm{mM}$ acetate produced (Supplementary Table 2). Thus, the final concentration of acetate was 1.5 times higher than the concentration, calculated from the amount of consumed ethanol. It could only be explained by additional acetate production from $\mathrm{CO}_{2}$ by G. ferrihydriticus.

Mössbauer analysis revealed that during bacterial growth, $\sim 10 \%$ of siderite was transformed into new phases, containing $\mathrm{Fe}$ (II) and $\mathrm{Fe}$ (III) atoms (Fig. 2b, middle column in the groups). Compared with the processing model of the control sample, the presence of two new doublets and a sextet can be noticed corresponding to green rust $\left(D_{1}, D_{2}\right)$ and magnetite $\left(S_{1}\right)$. The share of different new-formed minerals varied in different replicates (Fig. 4a, b show predominant formation of green rust and magnetite, respectively). The total intensity of the subspectra corresponding to $\mathrm{Fe}(\mathrm{III})$ atoms in the final precipitate was $\sim 4 \%$ (Supplementary Table 5). Summarily, the growth of the syntrophic co-culture on siderite led to its anaerobic oxidation into a mixture of magnetite and green rust. No structural changes of siderite appeared in sterile control (Fig. 4c).

No ethanol consumption, acetate production, or siderite transformations were observed in the control experiment with the heat-treated co-culture of $C$. "C. alkalaceticum" and G. ferrihydriticus. The pure culture of G. ferrihydriticus 
grew on the medium with ethanol and siderite producing 1-2 $\mathrm{mM}$ of acetate. At this, no ethanol oxidation was observed. Cell yield comprises $3.1 \pm 0.5 \times 10^{6}$ cells ml $^{-1}$. Growth of $G$. ferrihydriticus was accompanied by the same siderite color changes as in the experiment with syntrophic co-culture, and Mössbauer analysis revealed the formation of approximately $5 \%$ of magnetite. Thus, the growth of G. ferrihydriticus led to siderite oxidation in anaerobic conditions with production of acetate.

\section{Growth of C. "C. alkalaceticum" and G. ferrihydriticus co-culture on the mineral-free medium}

Active bacterial growth of the co-culture started in the mineral-free medium after 2 weeks of incubation and was accompanied by ethanol oxidation and acetate production (Figs. 1c, 2c and Supplementary Table 3). The medium remained transparent, but a thin layer of cream-colored flaky precipitate was formed on the bottom of the flasks (Supplementary Fig. 4). Direct cell-counting in the precipitate revealed the predominance of $C$. "C. alkalaceticum". The ratio of $C$. "C. alkalaceticum" to G. ferrihydriticus cells after 19 days of incubation comprises a 10:1 ratio, but at the end of the experiment, it changed to $3: 1$ due to the lysis of $C$. "C. alkalaceticum" cells (Fig. 1c and Supplementary Table. 3). SEM analysis of the flaky precipitate confirmed that it contained mostly the cells of $C$. "C. alkalaceticum", with rare cells of G. ferrihydriticus immersed in an extracellular polymeric matrix (Supplementary Fig. 5). The final ratio of $C$. "C. alkalaceticum" and G. ferrihydriticus cells in the co-culture growing on ethanol, detected by 16S rRNA gene fragment profiling, was 6:1 (Fig. 2c, last column in the group). At the end of the experiment, $14 \pm 2.9 \mathrm{mM}$ ethanol ( $41 \%$ of initial amount) was consumed and $22 \pm 1.6 \mathrm{mM}$ acetate was produced (Figs. 1c and 2c, first column in the group, and Supplementary Table. 3) being 1.6 times higher than the concentration calculated from the amount of consumed ethanol. The results of the experiment completely confirmed our suggestion about the ability of G. ferrihydriticus for hydrogenotrophic homoacetogenesis in the syntrophic association.

No growth on the medium with ethanol and without iron minerals was observed in the control experiment with the heat-treated co-culture. The pure culture of $G$. ferrihydriticus grew weakly $\left(1.0 \times 10^{6} \mathrm{cell} \mathrm{ml}^{-1}\right)$ in these conditions with the doubling time of 3.6 days, forming a dark, flaky precipitate on the bottom of the flasks (Supplementary Fig. 4) and producing only $2-5 \mathrm{mM}$ acetate within 30 days of incubation.

An additional experiment with molecular hydrogen was performed to prove the ability of G. ferrihydriticus to use hydrogen and carbonate for catabolic reaction. No growth with $100 \%$ and $25 \%$ hydrogen in the gas phase was detected, but the cells $\left(5 \times 10^{5} \mathrm{cell} \mathrm{ml}^{-1}\right)$ stayed alive and the production of $2 \mathrm{mM}$ acetate was registered.

Thus, though not growing by homoacetogenesis in the absence of iron minerals, the resting cells of G. ferrihydriticus were able to support the growth of $C$. "C. alkalaceticum" at these conditions, eliminating hydrogen formed in the course of ethanol oxidation.

\section{Discussion}

In this work, we studied syntrophic interactions between two alkaliphilic bacteria, oxidizing a non-fermentable substrate (ethanol) anaerobically. It was oxidized by the obligately syntrophic bacterium $C$. "C. alkalaceticum", while the released $\mathrm{H}_{2}$ was consumed by $G$. ferrihydriticus via $\mathrm{Fe}$ (III)-reduction, or hydrogenotrophic homoacetogenesis. Both these processes are supposed to be the most ancient types of microbial anaerobic respiration [40-42]. For alkaline carbonate-containing environments, such as soda lakes, the syntrophic interactions based on iron reduction or hydrogenotrophic homoacetogenesis so far have not been studied. Energy distribution between partner organisms in syntrophic communities from alkaline environments differ from that in neutrophilic conditions because IIET, as well as iron metabolism, are strongly $\mathrm{pH}$-dependent process [16, 17, 43]. The direct impact of $\mathrm{pH}$ is caused by the changes in proton chemical activities and applies to the reactions that consume or produce protons; indirect impact comes from $\mathrm{pH}$ control of the activities of chemical species involved in microbial reactions.

The results of our experiments with carbonate as the sole electron acceptor clearly demonstrate the ability of $C$. "C. alkalaceticum" and G. ferrihydriticus to oxidize ethanol in a syntrophic culture (Fig. 6). Gibbs-free energy changes, calculated for the reaction of ethanol oxidation, showed that it is favorable if component activities, alkaline $\mathrm{pH}$, and initial ethanol concentration are considered $\left(\Delta G^{\exp }{ }_{r}\right)$ :

$$
\begin{gathered}
\mathrm{CH}_{3} \mathrm{CH}_{2} \mathrm{OH}+\mathrm{H}_{2} \mathrm{O}=\mathrm{CH}_{3} \mathrm{COO}^{-}+\mathrm{H}^{+}+2 \mathrm{H}_{2} \\
\Delta G_{r}^{\circ}=+47.6 \mathrm{~kJ} / \mathrm{mol} ; \Delta G_{r}^{\exp }=-87.0 \mathrm{~kJ} / \mathrm{mol}
\end{gathered}
$$

Increased amount of acetate formed in our experiments indicates that hydrogenotrophic homoacetogenesis works as the driving force for ethanol oxidation (Figs. 1c and 2a, first column at each time point), but this proton-dependent reaction is less favorable in the given conditions than at the standard state:

$$
\begin{gathered}
4 \mathrm{H}_{2}+2 \mathrm{HCO}_{3}^{-}+\mathrm{H}^{+}=\mathrm{CH}_{3} \mathrm{COO}^{-}+4 \mathrm{H}_{2} \mathrm{O} \\
\Delta G_{r}^{\circ}=-139.8 \mathrm{~kJ} / \mathrm{mol} ; \Delta G_{r}^{\exp }=-49.6 \mathrm{~kJ} / \mathrm{mol}
\end{gathered}
$$


During syntrophic growth on the mineral-free medium, $12.7 \mathrm{mM}$ ethanol were utilized, and the economic coefficient for $C$. "C. alkalaceticum" comprises $9 \times 10^{6}$ cells per $1 \mathrm{mM}$ ethanol consumed (Fig. 1c and Supplementary Table 3). Key enzymes of acetogenesis coupled to Fe(II) oxidation have been identified in G. ferrihydriticus earlier [22], indicating the principal ability of the organism to grow using carbonate as the electron acceptor, reducing it to acetate. Though G. ferrihydriticus is able to grow lithotrophically, reducing ferrihydrite with molecular hydrogen [27], we failed to demonstrate its acetogenic growth with carbonate and 100 or $25 \%$ hydrogen as the electron donor. Either a lower concentration of hydrogen is necessary for this process (and this condition is realized during syntrophic growth), or it could grow by DIET from the cells of C. "C. alkalaceticum", similarly to the electrosynthesis process, in which microorganisms use electrons derived from a cathode of a bioelectrochemical system to reduce $\mathrm{CO}_{2}$ into acetate at neutral conditions [44, 45].

Microorganisms proliferate in the $E_{h}$-pH stability field of their metabolic products and metastability field of substrates [46]. Thus, the scenario of the syntrophic co-culture growth in the presence of iron-containing minerals is determined by the thermodynamic stability of these minerals. The fields of mineral stability designated on the $E_{h}$-pH diagram (Fig. 5) showed that all the reactions took place on the field of magnetite stability. Ferrihydrite is not stable, but siderite is stable at $\mathrm{pH}<6.5$ in a narrow Eh range. The initial (empty points) and final (filled points) $\mathrm{pH}$ and Eh values measured in the experiment lie in the field of magnetite stability (Fig. 5).

In the presence of metastable ferrihydrite, several reactions took place. G. ferrihydriticus started to grow, reducing ferrihydrite to the magnetically ordered phase according to the reaction:

$$
\begin{gathered}
\mathrm{CH}_{3} \mathrm{CH}_{2} \mathrm{OH}+12 \mathrm{Fe}(\mathrm{OH})_{3}=\mathrm{CH}_{3} \mathrm{COO}^{-}+4 \mathrm{Fe}_{3} \mathrm{O}_{4} \\
+\mathrm{H}^{+}+19 \mathrm{H}_{2} \mathrm{O} \\
\Delta G_{r}^{\circ}=-304.5 \mathrm{~kJ} / \mathrm{mol} ; \Delta G_{r}^{\mathrm{exp}}=-358.5 \mathrm{~kJ} / \mathrm{mol}
\end{gathered}
$$

Obviously, this reaction is more favorable in alkaline conditions. However, accumulation of magnetite on the surface of ferrihydrite could hinder G. ferrihydriticus from accessing $\mathrm{Fe}(\mathrm{III})$, and, thus, decrease the energy efficiency of ferrihydrite reduction. This process is likely to occur in our experiments, as follows from a slight decrease in G. ferrihydriticus cell numbers after 13 days of incubation (Fig. 1a). At the same time, the number of $C$. "C. alkalaceticum" cells increases, indicating the activation of syntrophic ethanol oxidation. Notably, besides hydrogenothrophic homoacetogenesis, G. ferrihydriticus is able to use $\mathrm{H}_{2}$ or acetate as the electron donors during ironreduction $[23,27]$. However, the calculated thermodynamic

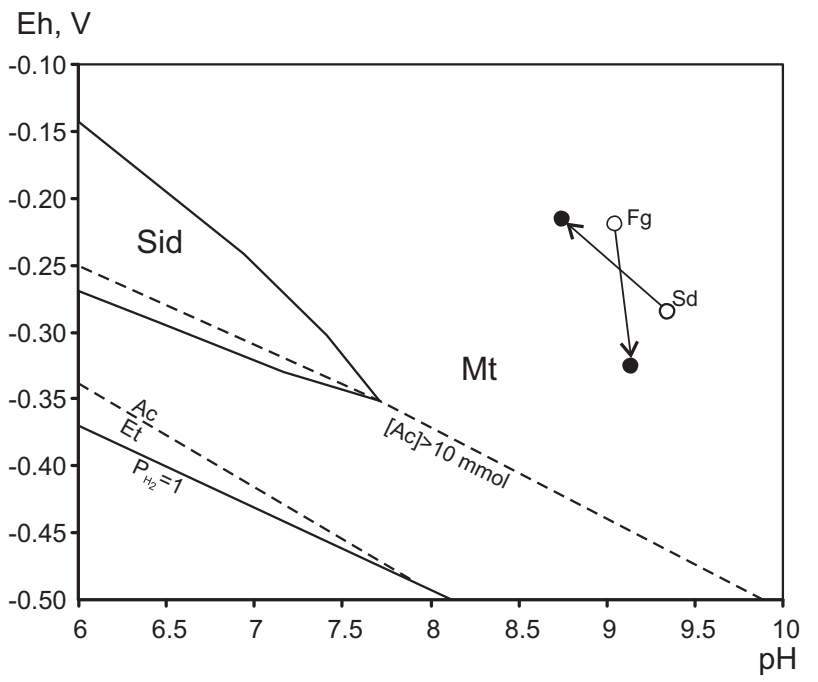

Fig. 5 Calculated Eh-pH diagram for the experimental system (initial $25 \mathrm{mM}$ ethanol, $75 \mathrm{mmol} \mathrm{Fe}$ ). Iron phases are shown by solid lines. Water stability is the solid line with $P_{\mathrm{H}_{2}}=1$. Organic matter concentrations are shown by dotted lines: $\mathrm{Ac} / \mathrm{Et}$-equilibrium concentrations of acetate and ethanol, [Ac] $>10 \mathrm{mM}-$ level of acetate concentration above which it become unstable. The initial and final conditions of experiments with ferrihydirte $(\mathrm{Fg})$ and siderite $(\mathrm{Sd})$ are shown with empty and filled circles, respectively. Sid—siderite; Mt—magnetite

equilibrium implies that further reduction of magnetite is favorable only in case of incomplete ethanol oxidation:

$$
\begin{gathered}
\mathrm{C}_{2} \mathrm{H}_{5} \mathrm{OH}+2 \mathrm{Fe}_{3} \mathrm{O}_{4}+6 \mathrm{HCO}_{3}^{-}+5 \mathrm{H}^{+} \\
=6 \mathrm{FeCO}_{3}+\mathrm{CH}_{3} \mathrm{COO}^{-}+7 \mathrm{H}_{2} \mathrm{O} \\
\Delta G_{r}^{\circ}=-309.1 \mathrm{~kJ} / \mathrm{mol} ; \Delta G_{r}^{\text {exp }}=-6.4 \mathrm{~kJ} / \mathrm{mol} \\
\mathrm{CH}_{3} \mathrm{COO}^{-}+4 \mathrm{Fe}_{3} \mathrm{O}_{4}+10 \mathrm{HCO}_{3}^{-}+11 \mathrm{H}^{+} \\
\quad=12 \mathrm{FeCO}_{3}+12 \mathrm{H}_{2} \mathrm{O} \\
\Delta G_{r}^{\circ}=-573.6 \mathrm{~kJ} / \mathrm{mol} ; \Delta G_{r}^{\exp }=+84.4 \mathrm{~kJ} / \mathrm{mol} \\
\mathrm{H}_{2}+\mathrm{Fe}_{3} \mathrm{O}_{4}+3 \mathrm{HCO}_{3}^{-}+3 \mathrm{H}^{+}=3 \mathrm{FeCO}_{3}+4 \mathrm{H}_{2} \mathrm{O} \\
\Delta G_{r}^{\circ}=-178.4 \mathrm{~kJ} / \mathrm{mol} ; \Delta G_{r}^{\text {exp }}=+45.8 \mathrm{~kJ} / \mathrm{mol}
\end{gathered}
$$

Results of the Mössbauer spectroscopy clearly showed that most of the siderite and green rust, that is, layered $\mathrm{Fe}$ (II)-Fe(III) hydroxicarbonate salts [47] formed at the end of the experiment, were the products of magnetite reduction (Fig. 2a, middle column of the groups; Fig. 3a, b; Supplementary Table 5). This transformation of magnetite could be explained by two equally probable processes. G. ferrihydriticus could either reduce magnetite, oxidizing ethanol to acetate, or use electrons produced by $C$. "C. alkalaceticum", via DIET through magnetite particles or conductive pili, also reducing magnetite (Fig. 6). The electron transfer strategy is also implies the capability of $C$. "C. alkalaceticum" to utilize magnetite as the electron acceptor instead of protons, which will be discussed below. Thus, after 13 days of cultivation and active ferryhydrite reduction 


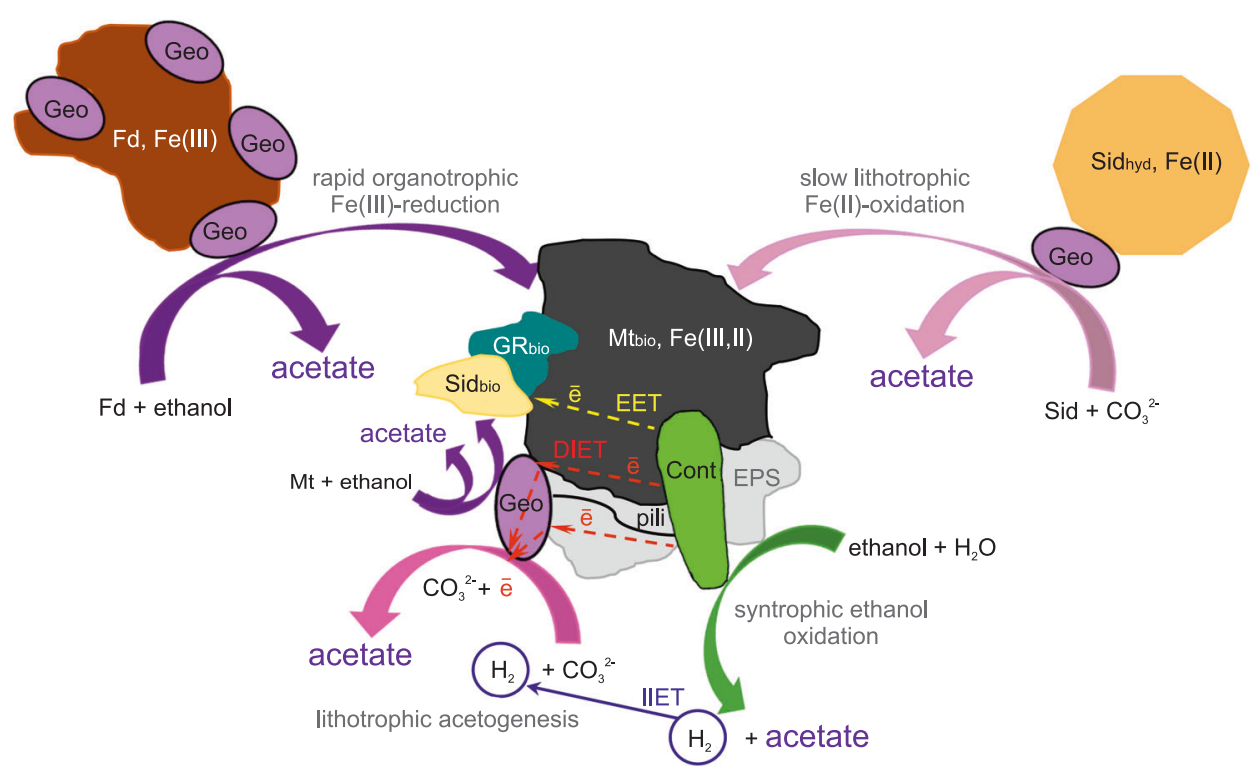

Fig. 6 Schematic representation of proposed pathways for interspecies interactions in the syntrophic co-culture of $C$. "C. alkalaceticum" and G. ferrihydriticus. Abbreviations: Geo-G. ferrihydriticus; Cont-C. "C. alkalaceticum"; $\mathrm{Fd}$-synthesized ferrihydrite; $\mathrm{Sid}_{\text {hyd }}$ - hydrothermal siderite; $\mathrm{Sid}_{\mathrm{bio}}$ - biogenic siderite; $\mathrm{Mt}_{\mathrm{bio}}$ - biogenic magnetite; $\mathrm{GR}_{\text {bio }}$ - biogenic green rust; EPS — extracellular polymeric substrate; EET-extracellular electron transfer; IIET-indirect interspecies

G. ferrihydriticus starts competing with $C$. "C. alkalaceticum" for both the electron donor (ethanol) and the electron acceptor (magnetite) (Fig. 1 and Supplementary Table 1).

Growth of the syntrophic co-culture with siderite was determined by three factors: thermodynamic instability of siderite at an alkaline $\mathrm{pH}$ (Fig. 5), properties of siderite surface, and the amount of active cells of $G$. ferrihydriticus inoculated in the flask. In the presence of siderite, G. ferrihydriticus cells could simultaneously perform hydrogenotrophic acetogenesis (reaction 2) and homoacetogenesis coupled to $\mathrm{Fe}$ (II) oxidation to magnetite:

$$
\begin{gathered}
12 \mathrm{FeCO}_{3}+12 \mathrm{H}_{2} \mathrm{O}=\mathrm{CH}_{3} \mathrm{COO}^{-}+4 \mathrm{Fe}_{3} \mathrm{O}_{4} \\
+10 \mathrm{HCO}_{3}^{-}+11 \mathrm{H}^{+} \\
\Delta G_{r}^{\circ}=+573.6 \mathrm{~kJ} / \mathrm{mol} ; \Delta G_{r}^{\exp }=-84.4 \mathrm{~kJ} / \mathrm{mol}
\end{gathered}
$$

Mössbauer spectroscopy revealed that about $10 \%$ of the siderite were oxidized by G. ferrihydriticus (Supplementary Table 4). The growth of G. ferrihydriticus in the presence of siderite was generally similar to that without iron minerals (Fig. 1b, c), but significant enhancement of $C$. "C. alkalaceticum" growth and almost complete ethanol oxidation were observed at these culture conditions (Fig. 1b). During the first 24 days of incubation, the rate of ethanol consumption was the same as in the mineral-free medium, i.e., $12.3 \mathrm{mM}$ ethanol were oxidized within this period and the economic coefficient for $C$. "C. alkalaceticum" comprises $8 \times 10^{6}$ cells per $1 \mathrm{mM}$ ethanol consumed (Fig. $1 \mathrm{~b}$, c and electron transfer; DIET - direct interspecies electron transfer. Figure legend: big arrows-chemical reactions provided by investigated microorganisms; dotted arrows-putative mechanisms of DIET between syntrophic partners: via electrically conductive magnetite or pili inside the EPS matrix. Red dotted arrows indicate putative DIET pathways, yellow dotted arrows indicate the proposed EET from C. "C. alkalaceticum" cells

Supplementary Tables 2 and 3). After the 24th day, cell numbers of the obligate syntroph increased and ethanol was completely consumed with fivefold higher economic coefficient of $43 \times 10^{6}$ cells per $1 \mathrm{mM}$ ethanol (Fig. 1b, and Supplementary Table 2). Considering the inability of $C$. "C. alkalaceticum" to grow in pure culture with ferrihydrite or siderite, the most probable explanation for its enhanced growth and metabolic activity on the medium with siderite could be the utilization of conductive magnetite produced by $G$. ferrihydriticus, as an additional external electron sink in the process of syntrophic ethanol oxidation. This implies the capability of $C$. "C. alkalaceticum" for extracellular electron transfer (EET) released during ethanol oxidation to magnetite (Fig. 6, yellow dotted arrow). It was previously shown that the addition of a small amount of magnetite as the conductive mineral facilitates syntrophic growth and methane production (see references in the review of refs. $[15,48,49])$. Thus, the observed magnetite formation during siderite oxidation by $G$. ferrihydriticus in a syntrophic co-culture may switch on EET from the obligate syntroph. It is worth mentioning that the growth of a syntrophic coculture in all variants was accompanied by an intensive production of EPS (Supplementary Figs. 3-5) that is regarded to play a significant role in EET [50, 51]. Further on, the presence of pili could be regarded as another means of possible way of electron transfer between the syntrophic partners. A homolog of electrically conductive pilin of $G b a$. sulfurreducens (e-pilin GSU1496) was previously identified 
in the G. ferrihydriticus genome [39]. Our alignment of a mature protein GSU1496 with a homologous mature pilin Ga0056053_00657 from G. ferrihydriticus, supported previous findings and revealed exact matches in 4 of 6 conserved positions of aromatic amino acids (Supplementary Fig. 6), which are regarded to determine electrontransferring properties of e-pili [52]. This fact together with our previous genomic analysis allow us to predict the production of highly conductive pili and external multiheme cytochromes by $G$. ferrihydriticus, although this feature needs further experimental evaluation.

\section{Conclusions}

Our study of syntrophic interactions in an alkaliphilic coculture oxidizing ethanol, which are summarized in Fig. 6, revealed that, in spite of the $\mathrm{pH}$ control of interspecies electron transfer via molecular hydrogen, alkaliphilic hydrogenotrophic homoacetogen ( $G$. ferrihydriticus) can provide the growth of the obligate syntroph $(C$. "C. alkalaceticum"), effectively utilizing $\mathrm{H}_{2}$ molecules or electrons released from ethanol oxidation even without active growth. This co-culture represents a novel combination of syntrophic partners connected by IIET and probably, DIET. The hydrogenotrophic partner organism can utilize the same organic substrate in a pure culture as the organotrophic partner. However, in the absence of an ferryhydrite as the most favorable electron acceptor $G$. ferrihydriticus performs homoacetogenesis in syntrophic association with $C$. "C. alkalaceticum". The presence of ferric or ferrous minerals, which could serve as the electron acceptors or donors for $G$. ferrihydriticus, respectively, did not destroy the syntrophic association. Both minerals used in our experiments are metastable at alkaline $\mathrm{pH}$ and were transformed by G. ferrihydriticus to the stable magnetite (Fig. 5). The utilization of ferrihydrite by $G$. ferrihydriticus as the electron acceptor for ethanol oxidation did not completely eliminate the obligate syntroph $C$. "C. alkalaceticum" from the co-culture, and, upon $\mathrm{Fe}(\mathrm{III})$ exhaustion, syntrophic ethanol oxidation resumed. Moreover, during organotrophic ferrihydrite reduction by $G$. ferrihydriticus, potential mediators of DIET between syntrophic partner organisms were formed (the conductive mineral magnetite, EPS matrix and pili). In its turn, complete ethanol oxidation and significant enhancement of $C$. "C. alkalaceticum" growth in the presence of the ferrous mineral siderite could be explained by the formation of magnetite, allowing consumption of electrons released from ethanol oxidation by $C$. "C. alkalaceticum" via EET mechanisms (Fig. 6). This allows us to propose that in anoxic sedimentary environments, complex interspecies redox interactions are established, depending on availability of ferrous or ferric iron minerals.
An important ecological implication of our results is the demonstration of the feasibility of syntrophic interactions based on redox transformation of iron minerals and lithotrophic homoacetogenesis. Our work clearly shows, that in the absence of methanogens and sulfate reducers such interaction could be realized. Syntrophic interactions via iron cycling discussed in the present work could play a significant role in Proterozoic biosphere, for which the predominance of iron cycle is well documented by the deposition of banded iron formations [53-55]. Moreover, it has been proposed that anoxic iron-rich and sulfatepoor marine conditions were common through most of the Earth history [56, 57]. Under such conditions, lithotrophic acetogenesis could work as an essential terminal reductive process, either for hydrogen-producing organisms or for $\mathrm{Fe}(\mathrm{II})$-oxidizers. In the latter case, coupling of acetogenesis with oxidation of Fe(II) minerals could explain yet poorly understood process of the formation of ferric minerals at anoxic conditions of the Precambrian period $[55,58]$.

Acknowledgements This work was supported by the Russian Science Foundation, project No. 17-74-30025 (cultivation experiments and iron minerals analysis), the Ministry of Science and Higher Education of the Russian Federation. Mössbauer analyses and thermodynamic calculations were performed with the support of the RFBR research projects No. 18-32-00349 and No. 18-05-00818 correspondingly.

\section{Compliance with ethical standards}

Conflict of interest The authors declare that they have no conflict of interest.

Publisher's note Springer Nature remains neutral with regard to jurisdictional claims in published maps and institutional affiliations.

\section{References}

1. Schink B. Synergistic interactions in the microbial world. Antonie Van Leeuwenhoek. 2002;81:257-61.

2. Bryant MP, Wolin EA, Wolin MJ, Wolfe RS. Methanobacillus omelianskii, a symbiotic association of two species of bacteria. Arch Microbiol. 1967;59:20-31.

3. Stams AJ, de Bok FA, Plugge CM, van Eekert MH, Dolfing J, Schraa G. Exocellular electron transfer in anaerobic microbial communities. Environ Microbiol. 2006;8:371-82.

4. Morris BE, Henneberger R, Huber H, Moissl-Eichinger C. Microbial syntrophy: interaction for the common good. FEMS Microbiol Rev. 2013;37:384-406.

5. Kouzuma A, Kato S, Watanabe K. Microbial interspecies interactions: recent findings in syntrophic consortia. Front Microbiol. 2015;6:477. https://doi.org/10.3389/fmicb.2015.00477.

6. Lovley DR, Fraga JL, Coates JD, Blunt-Harris EL. Humics as an electron donor for anaerobic respiration. Environ Microbiol. 1999;1:89-98.

7. Kaden J, Galushko A, Schink B. Cysteine-mediated electron transfer in syntrophic acetate oxidation by co-cultures of Geobacter sulfurreducens and Wolinella succinogenes. Arch Microbiol. 2002;178:53-8. 
8. Zhuang L, Ma J, Tang J, Tang Z, Shungui Z. Cysteine-accelerated methanogenic propionate degradation in paddy soil enrichment. Micro Ecol. 2016;73:916-24.

9. Overmann J, Schubert K. Phototrophic consortia: model systems for symbiotic interrelations between prokaryotes. Arch Microbiol. 2002;177:201-8.

10. Stams AJ, Oude Elferink SJ, Westermann P. Metabolic interactions between methanogenic consortia and anaerobic respiring bacteria. Adv Biochem Eng Biotechnol. 2003;81:31-56.

11. Stams AJ, Plugge CM, de Bok FA, van Houten BH, Lens P, Dijkman $\mathrm{H}$, et al. Metabolic interactions in methanogenic and sulfate-reducing bioreactors. Water Sci Technol. 2005;52:13-20.

12. Lovley DR. Syntrophy goes electric: direct interspecies electron transfer. Annu Rev Microbiol. 2017. https://doi.org/10.1146/a nnurev-micro-030117-020420.

13. Summers ZM, Fogarty H, Leang C, Franks AE, Malvankar NS, Lovley DR. Direct exchange of electrons within aggregates of an evolved syntrophic co-culture of anaerobic bacteria. Science. 2010;330:1413-5.

14. Rotaru A-E, Shrestha PM, Liu F, Shrestha M, Shrestha D, Embree $\mathrm{M}$, et al. A new model for electron flow during anaerobic digestion: direct interspecies electron transfer to Methanosaeta for the reduction of carbon dioxide to methane. Energy Environ Sci. 2014;7:408-15.

15. Kato S, Hashimoto K, Watanabe K. Methanogenesis facilitated by electric syntrophy via (semi)conductive iron-oxide minerals. Environ Microbiol. 2012;14:1646-54.

16. Jin Q, Kirk MF. $\mathrm{pH}$ as a primary control in environmental microbiology: 1. thermodynamic perspective. Front Environ Sci. 2018;6:21. https://doi.org/10.3389/fenvs.2018.00021.

17. Jin Q, Kirk MF. $\mathrm{pH}$ as a primary control in environmental microbiology: 2. kinetic perspective. Front Environ Sci. 2018;6:101. https://doi.org/10.3389/fenvs.2018.00101.

18. Zhilina TN, Zavarzina DG, Kolganova TV, Tourova TP, Zavarzin GA. Candidatus "Contubernalis alkalaceticum" an obligately syntrophic alkaliphilic bacterium capable of anaerobic acetate oxidation in a co-culture with Desulfonatronum cooperativum. Microbiology (Engl translation Mikrobiologiya). 2005;74:695-703.

19. Sorokin DY, Abbas B, Tourova TP, Bumazhkin BK, Kolganova TV, Muyzer G. (2014) Sulfate-dependent acetate oxidation at extremely natron-alkaline conditions by syntrophic associations from hypersaline soda lakes. Microbiology. 2014;160:723-32.

20. Zhilina TN, Zavarzina DG, Kuever J, Lysenko AM, Zavarzin GA. Desulfonatronum cooperativum sp. nov., a novel hydrogenotrophic, alkaliphilic, sulfate-reducing bacterium, from a syntrophic culture growing on acetate. Int J Syst Evol Microbiol. 2005;55:1001-6.

21. Sorokin DY, Abbas B, Geleijnse M, Kolganova TV, Kleerebezem $\mathrm{R}$, van Loosdrecht MC. Syntrophic associations from hypersaline soda lakes converting organic acids and alcohols to methane at extremely haloalkaline conditions. Environ Microbiol. 2016;18: 3189-202.

22. Zavarzina DG, Chistyakova NI, Shapkin AV, Savenko AV, Zhilina TN, Kevbrin VV, et al. Oxidative biotransformation of biotite and glauconite by alkaliphilic anaerobes: the effect of Fe oxidation on the weathering of phyllosilicates. Chem Geol. 2016;439: 98-109.

23. Zavarzina DG, Kolganova TV, Bouligina ES, Kostrikina NA, Tourova TP, Zavarzin GA. Geoalkalibacter ferrihydriticus gen. nov., sp. nov., the first alkaliphilic representative of the family Geobacteraceae, isolated from a soda lake. Microbiol (Engl translation Mikrobiologiya). 2006;78:723-31.

24. Yoho R, Popat S, Rago L, Guisasola A, Torres C. Anode biofilms of Geoalkalibacter ferrihydriticus exhibit electrochemical signatures of multiple electron transport pathways. Langmuir: ACS J Surf Colloids. 2015;31:12552-9.
25. Badalamenti JP, Krajmalnik-Brown R, Torres CI. Generation of high current densities by pure cultures of anode-respiring Geoalkalibacter spp. under alkaline and saline conditions in microbial electrochemical cells. mBio. 2013;4:e00144-13.

26. Badalamenti JP, Krajmalnik-Brown R, Torres CI, Bond DR. Genomes of Geoalkalibacter ferrihydriticus Z- $0531^{\mathrm{T}}$ and Geoalkalibacter subterraneus Red1 ${ }^{\mathrm{T}}$, two haloalkaliphilic metal-reducing Deltaproteobacteria. Genome Announc. 2015;3:e0039-15.

27. Zavarzina DG, Kevbrin VV, Zhilina TN, Chistyakova NI, Shapkin AA, Zavarzin GA. Reduction of synthetic ferrihydrite by a binary anaerobic culture of Anaerobacillus alkalilacustris and Geoalkalibacter ferrihydriticus grown on mannitol at $\mathrm{pH} 9.5$. Microbiology (English translation of Mikrobiologiya). 2011; 80: 743-57.

28. Chistyakova NI, Rusakov VS, Nazarova KA, Koksharov YuA, Zavarzina DG, Greneche J-M. Iron minerals formed by dissimilatory iron-and sulfur reducing bacteria studied by mossbauer spectrometry. Hyper Inter. 2008;182:55-63.

29. Kevbrin VV, Zavarzin GA. The effect of sulfur compounds on growth of halophilic the homoacetic bacterium Acetohalobium arabaticum. Microbiol (Engl translation Mikrobiologiya). 1992;61:812-7.

30. Stookey LL. Ferrozine-a new spectrophotometric reagent for iron. Anal Chem. 1970;42:779-81.

31. Matsnev ME, Rusakov VS. SpectrRelax: an application for Mössbauer spectra modeling and fitting. AIP Conf Proc. 2012; 1489:178-85.

32. Shvarov YV. HCh: new potentialities for the thermodynamic simulation of geochemical systems offered by Windows. Geochem Int. 2008;46:834-9.

33. Shvarov Y. A suite of programs, OptimA, OptimB, OptimC, and OptimS compatible with the Unitherm database, for deriving the thermodynamic properties of aqueous species from solubility, potentiometry and spectroscopy measurements. Appl Geochem. 2015;55:17-27.

34. Helgeson HC. Evaluation of irreversible reactions in geochemical processes involving minerals and aqueous solutions I. Thermodynamic relations. Geochim et Cosmoch Acta. 1968;32:853-77.

35. Lever MA, Torti A, Eickenbusch P, Michaud AB, Šantl-Temkiv T, Jørgensen BB. A modular method for the extraction of DNA and RNA, and the separation of DNA pools from diverse environmental sample types. Front Microbiol. 2015; May. https://doi. org/10.3389/fmicb.2015.00476.

36. Fadrosh DW, Ma B, Gajer P, Sengamalay N, Ott S, Brotman RM, et al. Animproved dual-indexing approach for multiplexed $16 \mathrm{~S}$ rRNA gene sequencing on the Illumina MiSeq platform. Microbiome. 2014;2:6.

37. Merkel AY, Pimenov NV, Rusanov II, Slobodkin AI, Slobodkina GB, Tarnovetckii IY, et al. Microbial diversity and autotrophic activity in Kamchatka hot springs. Extremophiles. 2017;21: 307-17.

38. Caporaso JG, Kuczynski J, Stombaugh J, Bittinger K, Bushman FD, Costello EK, et al. QIIME allows analysis of high-throughput community sequencing data. Nat Methods. 2010;7:335-6.

39. Holmes DE, Dang Y, Walker DJ, Lovley DR. The electrically conductive pili of Geobacter species are a recently evolved feature for extracellular electron transfer. Micro Genom. 2016;2:e00072.

40. Poehlein A, Schmidt S, Kaster A-K, Goenrich M, Vollmers J, Thürmer A, et al. An ancient pathway combining carbon dioxide fixation with the generation and utilization of a sodium ion gradient for ATP synthesis. PLoS ONE. 2012;7:e33439.

41. Martin WF, Sousa FL, Lane N. Evolution. Energy at life's origin. Science. 2014;344:1092-3.

42. Vargas M, Kashefi K, Blunt-Harris EL, Lovley DR. Microbiological evidence for $\mathrm{Fe}(\mathrm{III})$ reduction on early Earth. Nature. 1998;395:65-7. 
43. Straub KL, Benz M, Schink B. (2001) Iron metabolism in anoxic environments at near neutral pH. FEMS Microbiol Ecol. 2001;34:181-6.

44. Nevin KP, Woodard TL, Franks AE, Summers ZM, Lovley DR. Microbial electrosynthesis: feeding microbes electricity to convert carbon dioxide and water to multicarbon extracellular organic compounds. mBio. 2010;1. https://doi.org/10.1128/mBio.0010310 .

45. Nevin KP, Hensley SA, Franks AE, Summers ZM, Ou JH, Woodard TL, et al. Electrosynthesis of organic compounds from carbon dioxide is catalyzed by a diversity of acetogenic microorganisms. Appl Environ Microbiol. 2011;77:2882-6.

46. Zavarzin GA. The lithotrophyc microorganisms. Moscow: Nauka; 1972.

47. Legrand L, Mazerolles L, Chaussé A. The oxidation of carbonate green rust into ferric phases: solid-state reaction or transformation via solution. Geoch Cosm Acta. 2004;68:3497-507.

48. Viggi CC, Rossetti S, Fazi S, Paiano P, Majone M, Aulenta F. Magnetite particles triggering a faster and more robust syntrophic pathway of methanogenic propionate degradation. Environ Sci Technol. 2014;48:7536-43.

49. Fu L, Song T, Zhang W, Zhang J, Lu Y. (2018). Stimulatory effect of magnetite nanoparticles on a highly enriched butyrateoxidizing consortium. Front Microbiol. 2018;9:1480. https://doi. org/10.3389/fmicb.2018.01480.

50. Li S-W, Sheng G-P, Cheng Y-Y, Yu H-Q. Redox properties of extracellular polymeric substances (EPS) from electroactive bacteria. Sci Rep. 2016;6:390-8.
51. Xiao Y, Zhang E, Zhang J, Dai Y, Yang Z, Christensen H, et al. Extracellular polymeric substances are transient media for microbial extracellular electron transfer. Sci Adv. 2017;3: e1700623.

52. Walker DJF, Ramesh Y, Adhikari RY, Holmes DE, Ward JE, Woodard TL, et al. Electrically conductive pili from pilin genes of phylogenetically diverse microorganisms. ISME J. 2018;12: $48-58$.

53. Trendall AF. The significance of iron-formation in the Precambrian stratigraphic record. International Association of Sedimentologists. Spec Publ. 2002;33:33-66.

54. Klein C. Some Precambrian banded iron formations (BIFs) from around the world. Their age, geologic setting, mineralogy, metamorphism, geochemistry, and origin. Am Miner. 2005;90: 1473-99.

55. Konhauser KO, Planavsky NJ, Hardisty DS, Robbins LJ, Warchola TJ, Haugaard R, et al. Iron formations: a global record of Neoarchaean to Palaeoproterozoic environmental history. Earth Sci Rev. 2017;172:140-77.

56. Anbar AD, Duan Y, Lyons TW, Arnold GL, Kendall B, Creaser RA, et al. A whiff of oxygen before the Great Oxidation Event? Science. 2007;317:1903-6.

57. Poulton SW, Canfeld DE. Ferruginous conditions: a dominant feature of the ocean through Earth's history. Elements. 2011;7: 107-12.

58. Posth NR, Köhler I, Swanner ED, Schröder C, Wellmann E, Binder B, et al. (2013) Simulating Precambrian banded iron formation diagenesis. Chem Geol. 2013;362:66-73. 